\title{
Trace-space reconstruction of low-emittance electron beams through betatron radiation in laser-plasma accelerators
}

\author{
A. Curcio, ${ }^{1,2, *}$ M. Anania, ${ }^{1}$ F. Bisesto, ${ }^{1,2}$ E. Chiadroni, ${ }^{1}$ A. Cianchi, ${ }^{3}$ M. Ferrario, ${ }^{1}$ F. Filippi, ${ }^{1,2}$ \\ D. Giulietti, ${ }^{4}$ A. Marocchino, ${ }^{1}$ M. Petrarca, ${ }^{5}$ V. Shpakov, ${ }^{1}$ and A. Zigler ${ }^{1,6}$ \\ ${ }^{1}$ INFN-LNF, via Enrico Fermi 40, 00044 Frascati, Rome, Italy \\ ${ }^{2}$ Department of Physics, "Sapienza" University of Rome, Piazzale A. Moro 2, I-00185 Rome, Italy \\ ${ }^{3}$ INFN and Department of Physics, "Tor Vergata" University, \\ via della ricerca Scientifica 1, 00133 Rome, Italy \\ ${ }^{4}$ Physics Department of the University and INFN, Largo Bruno Pontecorvo 3, 56127 Pisa, Italy \\ ${ }^{5}$ Department of Basic and Applied Sciences for Engineering (SBAI) and INFN-Romal, \\ "Sapienza" University of Rome, Via A. Scarpa 14, 00161 Rome, Italy \\ ${ }^{6}$ Racah Institute of Physics, Hebrew University, Jerusalem 91904, Israel
}

(Received 16 September 2016; published 17 January 2017)

\begin{abstract}
A new methodology able to model and reconstruct the transverse trace space of low-emittance electron beams accelerated in the bubble regime of laser-plasma interaction is presented. The single-shot measurement of both the electron energy spectrum and the betatron radiation spectrum is shown to allow a complete measurement of the transverse emittance, including the correlation term. A novel technique to directly measure the betatron oscillation amplitude distribution is described and tested at the SPARC-LAB test facility through the interaction of the ultrashort ultraintense Ti:Sa laser FLAME with a He gas-jet target. Via the exposed technique the beam transverse profile is also retrieved. From the study of the electron transverse dynamics inside the plasma bubble, the nonlinear correlation between the betatron amplitude and the divergence, i.e. the angle with respect the acceleration axis, is found. The angular distribution of the electron beam inside the bubble is retrieved. The knowledge of the trace-space density allows a more accurate measurement of the transverse emittance with respect to previous paradigms.
\end{abstract}

DOI: 10.1103/PhysRevAccelBeams.20.012801

\section{INTRODUCTION}

The betatron radiation $[1,2]$ from laser-plasma accelerated electrons [3-5] has been largely studied both theoretically and experimentally. It has been used both as a collimated, bright [6-8] x-ray source for probing experiments [9] and as a diagnostic for plasma accelerated electrons [10-15]. In principle the incoherent spectrumangular distribution of the betatron radiation contains all the information about the emitting electron bunch while it is accelerated inside the plasma. The betatron spectrum is synchrotronlike [16] and its shape can be characterized by the so-called critical energy parameter [1]. The critical energy is related to the energy spectrum of the emitting electrons, to their spatial profile and lastly to the background plasma electron density. In this paper we present for the first time a method to measure the distribution function of the betatron oscillation amplitudes, which in previous works could be only simulated [10], relatively to an

*alessandro.curcio@lnf.infn.it

Published by the American Physical Society under the terms of the Creative Commons Attribution 4.0 International license. Further distribution of this work must maintain attribution to the author(s) and the published article's title, journal citation, and DOI. electron bunch accelerated in the bubble regime [17,18]. In this way the transverse profile of the electron beam, related to the betatron oscillation amplitude distribution, is retrieved. The to date mostly exploited transverse diagnostic techniques related to betatron radiation are based on $\mathrm{x}$-ray Fresnel diffraction $[12,13]$ by sharp metallic objects, leading to the measurement of the average electron beam radius inside the plasma [8] but not to the beam transverse profile. Even different techniques based on x-ray spectroscopic analysis of betatron radiation [14,15] led to the determination only of the average electron beam radius. An expression is given for the correlation function between the betatron oscillation amplitude and the divergence of the single accelerated electrons, i.e. the angle with respect the acceleration axis, in order to obtain the distribution of the electron divergences. This distribution is then compared to the one measured outside the plasma on the scintillator screen of a magnetic spectrometer. The reconstruction of the electron trace space is then performed, which allows a complete measurement of the transverse emittance, including the correlation term. The experiment was performed at the SPARC-LAB test facility [19] through the interaction of the ultrashort ultraintense Ti:Sa laser FLAME [19-22] with a He gas-jet target. We show that the single shot measurement of both the betatron spectrum and the electron energy spectrum is capable to provide all the information 
about the transverse trace space of the accelerated electrons. The final normalized emittance, measured with this novel and accurate technique, turns out to be a fraction of the upper limit evaluated by the product of the beam average energy times the beam radius times the beam divergence $[10,11]$, since the correlation term can be also taken into account.

\section{DESCRIPTION OF THE METHOD}

The definition of geometric radial emittance $\epsilon_{r}$ of an accelerated beam, which is related to the area occupied by electrons in the $\left(r, \theta_{d}\right)$ trace space, where $r$ is the transverse displacement and $\theta_{d}=d r / d z$ is the transverse angle with respect to the propagation axis $z$, is given by [23]

$$
\epsilon_{r}=\sqrt{\left\langle\Delta r^{2}\right\rangle\left\langle\Delta \theta_{d}^{2}\right\rangle-\left\langle\Delta r \Delta \theta_{d}\right\rangle^{2}},
$$

where $\Delta r=r-\langle r\rangle$ and $\Delta \theta_{d}=\theta_{d}-\left\langle\theta_{d}\right\rangle$. The averages denoted by angular brackets in Eq. (1) are considered with respect to the electron trace-space density, which takes into account how many electrons occupy an elemental area in the trace space. In the following we are going to introduce a method to model and reconstruct the trace-space density starting from the beam characteristic functions $P(r)$ and $\Theta\left(\theta_{d}\right)$, i.e. the beam profile and the distribution of the singleelectron divergences respectively, and the correlation function $r\left(\theta_{d}\right)$ which links the betatron oscillation amplitude to the divergence of the single electrons, so allowing the computation of the correlation term in Eq. (1) (the second term under the square root). The method consists, as will be shown, in measuring $R\left(r_{\beta}\right)$, the distribution of the betatron oscillation amplitudes $r_{\beta}$, then in retrieving $P(r)$ and $\Theta\left(\theta_{d}\right)$ from the simultaneous detection of the betatron radiation spectrum and the electron energy spectrum. In fact the distribution $R\left(r_{\beta}\right)$ contains information about the electron beam profile: by generalizing Eq. (78) of Ref. [24] to a generic profile, the beam radial profile $P(r)$ and the function $R\left(r_{\beta}\right)$ can be related through Eq. (2):

$$
R(r)=r P(r),
$$

where we identified $r=r_{\beta} / \sqrt{2}$. This result comes by considering the electron trajectories of Ref. [24], i.e. $r(z)=r_{\beta} \cos \left[k_{\beta} z+\phi_{i}\right]$, where $\phi_{i}$ is the initial phase and $k_{\beta}$ is the betatron wave number, for which the rms value of $r$ over a period $2 \pi / k_{\beta}$ is $r_{\beta} / \sqrt{2}$. Therefore from the function $R\left(r_{\beta}\right)$ is possible to retrieve the radial beam profile $P(r)$ via Eq. (2). The single particle emission model for the betatron radiation, following Ref. [24], gives

$S_{\gamma, r_{\beta}}(E, \Omega)=\sum_{n} \frac{\alpha N_{\beta}^{2} \gamma^{2} E R_{n}}{2 \hbar \omega_{\beta}}\left[C_{r}^{2}+C_{z}^{2} \theta^{2}-2 C_{r} C_{z} \theta \cos \phi\right]$, where $S_{\gamma, r_{\beta}}=d^{2} I / d E d \Omega$ is the radiated energy $d I$ per unit photon energy $d E$ and solid angle $d \Omega=\sin \theta d \theta d \phi, \alpha$ is the fine structure constant, $N_{\beta}$ is the number of betatron oscillations, $\gamma$ is the Lorentz factor of the emitting electron, $E$ is the energy of the emitted photon, $\omega_{\beta}=\omega_{p} / \sqrt{2 \gamma}$ is the betatron frequency related to the plasma frequency $\omega_{p}=\sqrt{n_{e} e^{2} / m \varepsilon_{0}}$, where $n_{e}$ is the electron plasma density, $m$ is the electron mass, $e$ is the elementary charge and $\varepsilon_{0}$ is the vacuum dielectric constant. The summation in Eq. (3) runs over $n$, the harmonic numbers of the resonance function $R_{n}$. We have defined

$$
\begin{gathered}
C_{r}=r_{\beta} k_{\beta} \sum_{m} J_{m}\left(\rho_{z}\right)\left[J_{n+2 m-1}\left(\rho_{r}\right)+J_{n+2 m+1}\left(\rho_{r}\right)\right] \\
C_{z}=\sum_{m} 2 J_{m}\left(\rho_{z}\right) J_{n+2 m}\left(\rho_{r}\right) \\
\rho_{z}=\frac{E r_{\beta}^{2} k_{\beta}^{2}}{8 \hbar \omega_{\beta}} ; \quad \rho_{r}=\frac{E r_{\beta} k_{\beta} \theta \cos \phi}{\hbar \omega_{\beta}} \\
R_{n}=\operatorname{sinc}^{2}\left[\frac{\pi N_{\beta}}{2 \gamma^{2} \hbar \omega_{\beta}}\left(E-\frac{2 n \gamma^{2} \hbar \omega_{\beta}}{1+K_{\beta}^{2} / 2+\gamma^{2} \theta^{2}}\right)\right] .
\end{gathered}
$$

$J_{n}$ is the first kind $n$th order Bessel function and we have introduced the betatron strength parameter $K_{\beta}=\gamma r_{\beta} k_{\beta}$. The emission from a bunch of electrons [Eq. (8)] is the incoherent sum of the single-particle spectra [Eq. (3)], because in the case of betatron radiation from plasma accelerated electrons the length of the electron bunch is typically much larger than the radiated wavelength:

$$
S(E)=\iiint d \Omega d \gamma d r_{\beta} \Gamma(\gamma) R\left(r_{\beta}\right) S_{\gamma, r_{\beta}}(E, \Omega),
$$

where $\Gamma(\gamma)$ is the energy spectrum of the electrons. Let us approximate the integral over the betatron oscillation amplitudes in Eq. (8) with a summation (operation which is allowed for smooth and square integrable functions):

$$
\int d r_{\beta} R\left(r_{\beta}\right) \sim \sum_{i} R\left(r_{\beta, i}\right) \equiv \sum_{i} R_{i}
$$

where $r_{\beta, i}$ is the $i$ th betatron oscillation amplitude chosen in some range of values $\left[0, r_{\beta}^{\max }\right]$ and $R_{i}$ is proportional to the number of electrons which oscillate with that amplitude. The continuous and discrete distributions $R\left(r_{\beta}\right)$ and $R_{i}$ have not the same physical dimension because of a different normalization but they have the same physical meaning of betatron oscillation amplitude distributions. Equation (8) becomes

$$
S(E)=\sum_{i} R_{i} \iint d \Omega d \gamma \Gamma(\gamma) S_{\gamma, r_{\beta, i}}(E, \Omega) .
$$

Now let us consider a measured betatron spectrum $\Sigma(E)$ which will be in general, due to the limitation given by the 
spectral resolution intrinsic to the measurement, a vector $\Sigma\left(E_{j}\right) \equiv \Sigma_{j}$ where the $j$ index gives the $j$ th point in the detectable range. We can think to discretize also the theoretical spectrum described by Eq. (10) with respect to the photon energies with the same resolution of the measurement, so obtaining

$$
S\left(E_{j}\right) \equiv S_{j}=\sum_{i} R_{i} S_{i j},
$$

where we defined $S_{i j}=\iint d \Omega d \gamma \Gamma(\gamma) S_{\gamma, r_{\beta, i}}\left(E_{j}, \Omega\right)$. Finally, imposing the theoretical spectrum and the measured one to be equal, we obtain

$$
S_{j}-\Sigma_{j}=\sum_{i j} R_{i} S_{i j}-\Sigma_{j}=0 .
$$

The matrix $S_{i j}$ will be square and invertible only when the vector $R_{i}$, which is the solution of the linear system (12), has the same order of the vector $\Sigma_{j}$ and when the matrix determinant is different from zero. The first condition limits the resolution in the measurement of the betatron oscillation amplitude distribution. The second has to be valid in order to use the inversion procedure on the system of linear equations (12), nevertheless the spectrum model (3) is such that the corresponding matrix $S_{i j}$ is never singular. The system (12) states that once the electron energy spectrum $\Gamma(\gamma)$ is known and a model for the betatron radiation emitted by a single particle is chosen [Eq. (3)], the matrix $S_{i j}$ is calculable, at least numerically. Therefore, given a measurement of the betatron spectrum $\Sigma_{j}$, corresponding to an electron bunch with energy spectrum $\Gamma(\gamma)$, the inversion of the linear system (12) yields the solution vector $R_{i}$, which is the quantity we are interested in. It is worth noting that the validity of the present model, based on Eq. (3), is limited to cases when there is a preferential oscillation axis for the betatron motion or radial symmetry. Moreover, only the radiation corresponding to the last phase of the acceleration is considered, where the electron energy gain is maximum, being the radiation power also maximum in that point $[11,24]$. The choice of the spectrum model described by Eq. (3) is coherent with this last assumption. For $K_{\beta} \gg 1$ the spectrum in Eq. (3) reduces to a more handy synchrotronlike form [24], nevertheless, being the betatron strength parameter $r_{\beta}$-dependent, the asymptotic limit of synchrotron radiation emission cannot be applied to all the particles of the electron beam. Therefore the use of a general nonasymptotic expression for the emitted spectrum is necessary in order to reliably represent the single particle emission in each case. In the present work we do not consider the cases of Direct Laser Acceleration (DLA) [25], i.e. we focus on the cases when the laser pulse does not significantly extend inside the plasma accelerating structure and it does not directly interact with the accelerated electrons. The condition for this to be valid is $R>c \sigma_{\tau}$, where $R$ is the plasma bubble radius and $\sigma_{\tau}$ is the rms duration of the laser pulse.
The case of DLA will be faced in a dedicated future work, by starting from the DLA-modified betatron trajectories reported in [26]. In the next section the above inversion method is applied to a set of experimental data in order to measure the $R\left(r_{\beta}\right)$ distribution. From that we are going to retrieve the functions $P(r)$ through Eq. (2) and finally $\Theta\left(\theta_{d}\right)$ through the method we discuss in the following. In principle the function $\Theta\left(\theta_{d}\right)$ could be rather tricky to be measured because it is related to the electron bunch inside the plasma bubble where intercepting diagnostics cannot be used [27-31]. On the other hand it has been shown in previous works $[10,11]$ that the measurement of the divergence outside the plasma can be reliable when the effect of the vacuum-plasma interface and of the space-charge effects are negligible. Nevertheless, in the most general case, an electron bunch can undergo divergence modification at the exit of the plasma and a general technique to measure the divergence inside the bubble could be necessary. The divergence measured outside can be even underestimated when the plasma ramp at the vacuum-plasma interface is not sharp [31]. It can happen that $\Theta\left(\theta_{d}\right)$ is asymmetric in the two transverse planes. When it is measured on the scintillator screen of a magnetic spectrometer, it corresponds only to one plane, usually spanned by the laser wave vector and the magnetic field. On the other hand, the betatron oscillations can often occur in one plane, that of a p-polarized laser, orthogonal to the magnetic field lines. Therefore in some special case it could be possible to combine the measurement of the divergence outside the plasma with the one retrieved from betatron radiation analysis, in order to get the divergence in the two orthogonal planes. We aim to propose a method to retrieve the function $\Theta\left(\theta_{d}\right)$ inside the plasma bubble once the function $P(r)$ is known. Starting from the trajectories of Ref. [24], the electron divergence is calculated as

$$
\theta_{d}=\sqrt{\left\langle\left(\frac{d r}{d z}\right)^{2}\right\rangle-\left\langle\left(\frac{d r}{d z}\right)\right\rangle^{2}}=\frac{\sqrt{2}}{2} r_{\beta} k_{\beta}
$$

which is consistent with the expression reported in Ref. [11], except for the factor $1 / \sqrt{2}$ which comes after averaging over all the possible electron initial oscillation phases $\phi_{i}$. Even when using electron trajectories which take into account the electron acceleration [23,32], as $r(z)=r_{\beta}[\gamma(z)] \cos \left\{k_{\beta}[\gamma(z)] z+\phi_{i}\right\}$, the derivative $d r / d z$ consists of two classes of terms, the first dependent on the derivatives $d r_{\beta} / d z$ and the second dependent on the product $r_{\beta} d\left(k_{\beta} z\right) / d z$ :

$$
\frac{d r}{d z}=\cos \left[k_{\beta} z+\phi_{i}\right] \frac{d r_{\beta}}{d z}-r_{\beta} \frac{d\left(k_{\beta} z\right)}{d z} \sin \left[k_{\beta} z+\phi_{i}\right] .
$$

Therefore in this case also the divergence $\theta_{d}$ will consist of these two classes of terms. The first class describes the adiabatic focusing of the betatron oscillation amplitude, usually giving a negligible contribution to $\theta_{d}$ on the $\mu \mathrm{rad}$ 
scale. The second class of terms instead is related to the fast betatronic motion itself and is of the order $r_{\beta} k_{\beta}$ like in Eq. (13), giving a contribution on the mrad scale. For $\gamma \sim \gamma_{0}=\gamma_{\|}$, i.e. equating the electron energy to the longitudinal momentum, the correlation function $r\left(\theta_{d}\right)$ which derives from Eq. (13) would result in a zero emittance, being $k_{\beta}$ independent of $r_{\beta}$ and the correlation between the betatron oscillation amplitude and the divergence exactly linear. Therefore the anharmonicity of the betatron relativistic oscillations has to be taken into account. In fact the betatron motion can be compared to a wigglerlike oscillation. The effective electron Lorentz factor $\gamma^{*} \sim$ $\gamma_{0} / \sqrt{1+K_{\beta 0}^{2} / 2}$ has to be introduced, which considers the mass modification due to the relativistic transverse motion [33]. The factor $\gamma^{*}$ comes directly out when considering, like in Ref. [24], longitudinal trajectories oscillating at the double betatron frequency of the type $z=z_{0}+v_{z 0}\left(t-k_{\beta}^{2} r_{\beta}^{2} t / 4-k_{\beta}^{2} r_{\beta}^{2} \sin 2 \omega_{\beta} t / 8 \omega_{\beta}\right)$, where $z_{0}$ and $v_{z 0}$ are the electron initial longitudinal position and velocity respectively. When the parameter $K_{\beta 0}$ starts to be greater than one the anharmonicity in the electron motion cannot be neglected anymore because the average longitudinal velocity can be smaller than the instantaneous one [33]. We defined $K_{\beta 0}=\gamma_{0} k_{\beta 0} r_{\beta}$, where $k_{\beta 0}=k_{p} / \sqrt{2 \gamma_{0}}$ and $k_{p}=\omega_{p} / c$ is the plasma wave number with $c$ the velocity of light in vacuum. The presence of the transverse mass term $\sqrt{1+K_{\beta 0}^{2} / 2}$ ensures a realistic estimation of the transverse emittance. When the energy spread of the electron bunch is small $\left(\sigma_{\gamma} / \gamma_{0} \ll 1\right.$, with $\sigma_{\gamma}$ the standard deviation from the average energy $\gamma_{0}$ ), we can write $\theta_{d} \sim r_{\beta} k_{\beta}^{*} / \sqrt{2}$ committing an error of order $\sigma_{\gamma} / 2 \gamma_{0}$, where $k_{\beta}^{*}=k_{p} / \sqrt{2 \gamma^{*}}$. It is worth noting that in what was discussed so far the energy spread does not need to be constant in the entire acceleration process, because of the fact that we only consider the electron dynamics and the radiation in one instant of the process, when the electron energy gain is maximum. In this way the explicit nonlinear correlation between the betatron radius $r_{\beta}$ and the divergence $\theta_{d}$ through Eq. (13) is found:

$$
\theta_{d}=\sqrt{\frac{\sqrt{1+\frac{1}{2} \gamma_{0}^{2} r_{\beta}^{2} k_{\beta 0}^{2}}}{4 \gamma_{0}}} r_{\beta} k_{p} .
$$

This means that, after solving Eq. (15) with respect to $r_{\beta}=\sqrt{2} r$ (a sextic equation, analytically solvable), the function $\Theta\left(\theta_{d}\right)$ can be written as

$$
\Theta\left(\theta_{d}\right) \propto P\left[r\left(\theta_{d}\right)\right] .
$$

\section{DESCRIPTION OF THE EXPERIMENT}

A self-injection experiment was performed at the SPARC-LAB test facility (INFN-LNF) by using the laser
FLAME [19-22]. The main pulse characteristics were $\sim 1 \mathrm{~J}$ energy, delivered in $30 \mathrm{fs}$ (FWHM) over a $10 \mu \mathrm{m}$ diameter focus, corresponding to a laser strength parameter [4] $a_{0} \sim 4.4$. The laser was focused on a He gas-jet target (Fig. 1). The electron density of the generated plasma was measured through a Mach-Zender interferometer to be $n_{e}=(8 \pm 1) \times 10^{18} \mathrm{~cm}^{-3}$. The maximum acceleration length was $L_{a c c} \sim z_{d} \sim 1.2 \mathrm{~mm}$, where $z_{d}=4 c \sqrt{a_{0}} \omega_{0}^{2} / 3 \omega_{p}^{3}=$ $1.15 \mathrm{~mm}$ is the dephasing length $[4,18]$, with $\omega_{0}$ the laser pulsation, so that the electrons were accelerated up to the dephasing point. The extension of the laser-generated plasma at density $n_{e}$ is as long as several Rayleigh lengths. The FLAME laser contrast is reported in Ref. [34], showing that the intensity of the Amplified Spontaneous Emission (ASE) is $10^{-9}$ times the FLAME peak intensity, while the ps pedestal is $10^{-3}$. The high contrast between the ASE and the main pulse ensures that the ASE does not have an important effect in producing a preplasma, while the ps pedestal is not negligible. Nevertheless it has been shown that the ps pedestal can be responsible for the onset of optical guiding of the main pulse, determining the production of good quality electron beams [35]. This can justify together with the relativistic self-focusing effect [4] the relatively long high-intensity propagation distance of the laser pulse. The estimated bubble radius [18] $R=$ $2 \sqrt{a_{0}} c / \omega_{p} \sim 9 \mu \mathrm{m}$ was larger than $\sigma_{\tau} \sim 3.8 \mu \mathrm{m}$, so DLA effects could be neglected. The energy spectrum of the electrons was measured by using of a magnetic dipole coupled to a scintillator screen. The betatron radiation emitted by the accelerated electrons during the interaction of the high-intensity laser with the He plasma was detected

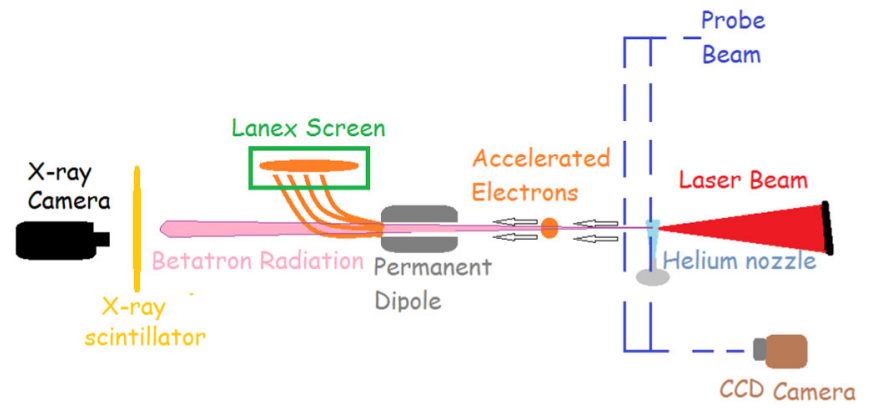

FIG. 1. The FLAME laser beam is focused on the He gas jet. Electrons are accelerated in the blowout regime over a distance $\gtrsim 1$. The permanent dipole $(\lesssim 1 \mathrm{~T}$ ) coupled with a scintillator screen allows the measurement of the electron energy spectrum. The x-ray betatron radiation, directed in the same direction of the impinging laser, crosses an $\mathrm{x}$-ray scintillator in order to detect the spatial distribution of the radiation and then it arrives at the x-ray spectrometer, where the photon spectrum is detected. Filters of kapton and aluminum are exploited on the light line in order to damp the x-ray radiation and screen the residual laser light. A probe beam is coupled to a Mach-Zender interferometer in order to retrieve the electron plasma density of the laser-produced plasma. 


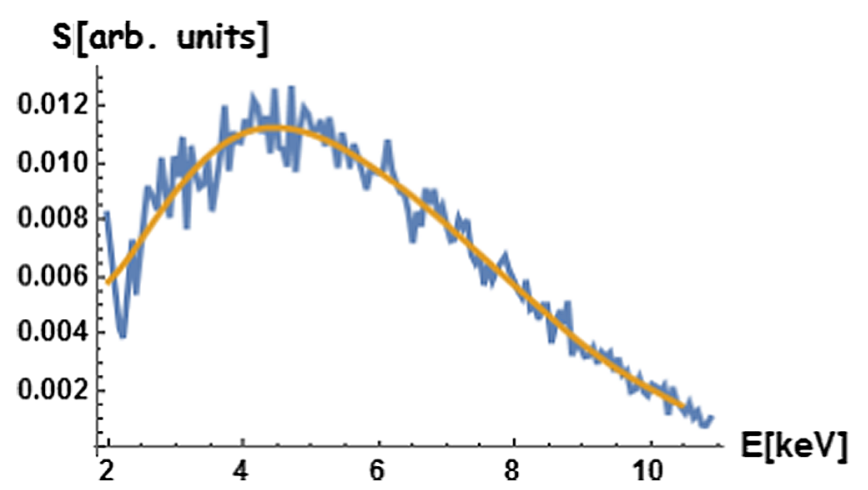

FIG. 2. Betatron spectrum detected with a CCD-X camera working in single photon counting mode together with a polynomial fit.

simultaneously both by an X-ray scintillator and a CCD-X camera calibrated during a previous experiment [36], positioned at $0.7 \mathrm{~m}$ from the interaction point, cooled down to $-30^{\circ} \mathrm{C}$ and shielded by a lead cage from broad-divergence bremsstrahlung. The goal was that of measuring simultaneously the spatial distribution of the radiation with the $\mathrm{x}$-ray scintillator and the spectrum in single photon counting [15] mode with the CCD-X camera. The X-ray scintillator was positioned in front of the CCD-X at $0.5 \mathrm{~m}$ from the interaction point. The betatron $\mathrm{x}$-ray flux had to cross an overall absorbing thickness constituted by a $280 \mu \mathrm{m}$ kapton window, a $200 \mu \mathrm{m}$ x-ray scintillator screen and $1 \mu \mathrm{m} \mathrm{Al} \mathrm{filter}$ to screen the residual infrared light on the camera. In Fig. 2 the betatron spectrum, i.e. the deconvolution of the signal detected by the CCD-X camera, is reported together with a polynomial fit, corresponding to the radiation emitted by the electron beam whose characteristics are shown in Fig. 3. In order to speed up and facilitate the convergence of the

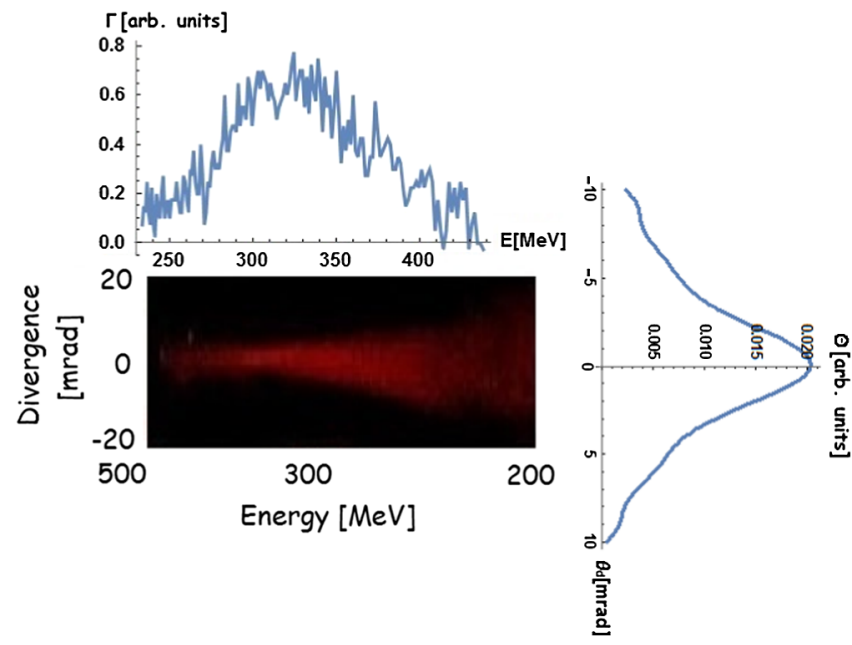

FIG. 3. Electron energy spectrum relative to the electron bunch accelerated in the bubble regime. Central energy $\gamma_{0}=320 \mathrm{MeV}$, energy spread $2 \sigma_{\gamma} / \gamma_{0} \sim 20 \%$. The outlines of the energy spectrum (up) and of the beam divergence (right) are reported. inversion procedure introduced before, the polynomial fit is exploited to construct the vector $\Sigma_{j}$ rather than the signal itself. This approach is justified if thinking that the fluctuations observed on the detected spectrum are not related to modulations of the betatron oscillation amplitude distribution function, but only to noise. In this way no essential physical information is lost about the function $R(r)$. The measured electron energy spectrum is shown in Fig. 3, corresponding to an average energy $\gamma_{0}=320 \mathrm{MeV}$ and to an energy spread $2 \sigma_{\gamma} / \gamma_{0} \sim 20 \%$. The energy spectrum represented in Fig. 3 is integrated over the detected distribution of the electron divergences in the vertical plane. The electron divergence distribution in the same plane is also reported, integrated over all the detected electron energies. We apply the inversion procedure described in the previous section, starting from the betatron spectrum of Fig. 2 and the electron spectrum of Fig. 3. The measured $R(r)$ function is reported in Fig. 4, together with the beam profile retrieved via Eq. (2). The errors on $R(r)$ and $P(r)$ curves have been estimated propagating the error on the electron plasma density measurement, taking into account the relative weight of the fluctuations of the detected betatron spectrum with respect to the polynomial fit and the error on the measurement of the electron average energy $\gamma_{0}=640 \pm 6$, the last determined by the error on the alignment of the dipole with respect to the main laser beam line and of the scintillator screen with respect to the magnet. The dotted lines in Fig. 4 identify the error range for both $R(r)$ and $P(r)$ and they represent the solutions obtained with the inversion code based on the system of equations (12) when the maximal and minimal values for electron plasma density, betatron spectrum and electron average energy according to the errors have been considered. The retrieved beam profile is not perfectly Gaussian. The measured rms betatron radius is $\sigma_{r}=$ $0.42 \pm 0.04 \mu \mathrm{m}$, in agreement with 3D PIC simulations performed with the ALADYN code [37-39], from which $\sigma_{r}^{\text {PIC }} \sim 0.4 \mu \mathrm{m}$. The simulations were performed for a driver laser pulse and a background electron plasma density with the same characteristics of the experimental ones. The code predicted a polyenergetic electron beam with central energy

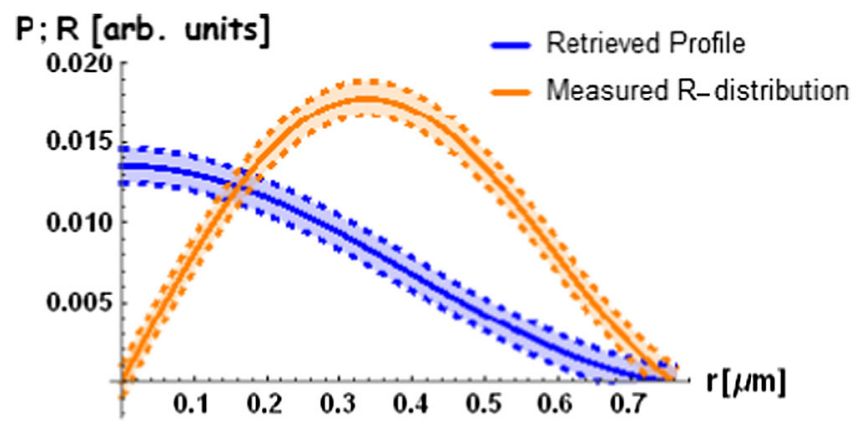

FIG. 4. The beam profile $P(r)$ derived directly from the measured function $R(r)$ via Eq. (2). The rms betatron radius is found $\sigma_{r} \sim 0.42 \pm 0.04 \mu \mathrm{m}$. 

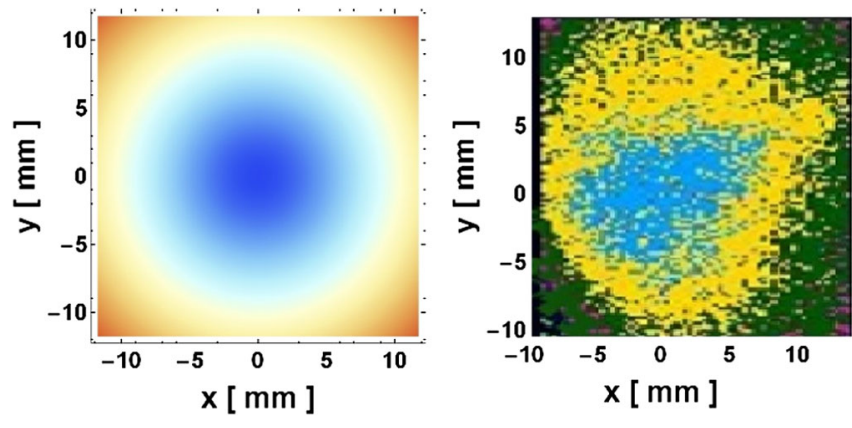

FIG. 5. Betatron radiation spatial distribution: simulation (left) and measurement (right). Measured divergence of the radiation beam $\theta_{\beta}^{\text {meas }}=8.2 \pm 0.3 \mathrm{mrad}$, simulation value $\theta_{\beta}^{\text {sim }}=8.5 \mathrm{mrad}$.

$\sim 320 \mathrm{MeV}$, consistent both with the measurement and with the expression given by Ref. [18], from which the maximum electron energy gain in the bubble regime is $\gamma_{\max } \sim$ $2 a_{0} \omega_{0}^{2} / 3 \omega_{p}^{2}$. The retrieved beam size value $\sigma_{r}$ is not only cross-checked through the 3D PIC simulation but also through the analysis of the betatron radiation spatial distribution. In fact the betatron radiation divergence $\theta_{\beta}$ satisfies the relation $\theta_{\beta} \sim \sigma_{r} k_{\beta 0}$ [8]. In Fig. 5 the comparison is shown between the simulated and detected spatial distribution of betatron radiation. The radiation spatial distribution shows a radial symmetry. The simulation is performed considering an electron bunch with the detected properties, namely the radial distribution of Fig. 4 and the energy spectrum of Fig. 3. There is agreement, being the measured divergence of the radiation beam $\theta_{\beta}^{\text {meas }}=8.2 \pm 0.3 \mathrm{mrad}$ while the simulation value is $\theta_{\beta}^{\text {sim }}=8.5 \mathrm{mrad}$. Therefore the analysis of the spatial distribution confirms the validity of the value $\sigma_{r}=0.42 \mu \mathrm{m}$. In Fig. 6 the comparison between the $\Theta\left(\theta_{d}\right)$ function retrieved by exploiting the equations (2), (13), (15), (16) and the $\Theta\left(\theta_{d}\right)$ function measured outside the plasma (Fig. 3), is reported. The ramp at the vacuum-plasma interface was only a fraction of the effective betatron wavelength $\lambda_{\beta}^{*}=$ $2 \pi c\left(1+K_{\beta 0}^{2} / 2\right)^{1 / 4} / \sqrt{2 \gamma_{0}} \omega_{p}\left(L_{\mathrm{ramp}} \sim 100 \mu \mathrm{m} \ll \lambda_{\beta}^{*} \sim 600 \mu \mathrm{m}\right)$,

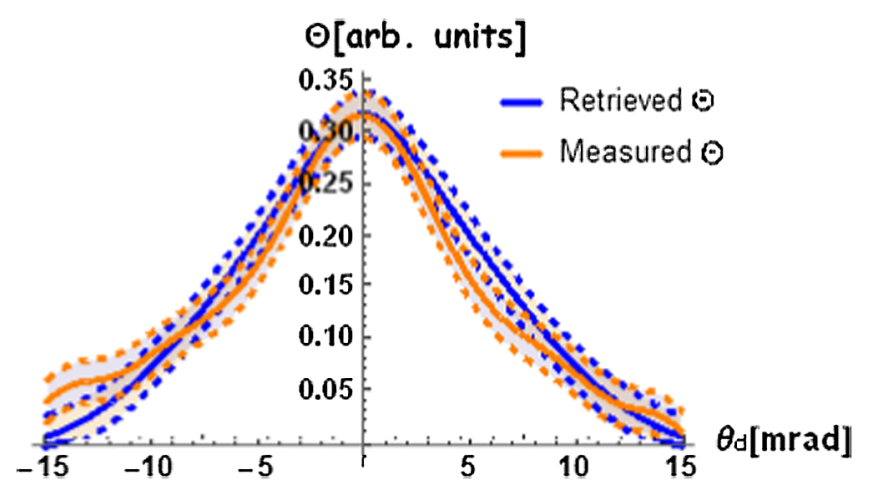

FIG. 6. Comparison between the measured (outside the bubble) and the retrieved (inside the bubble) $\Theta\left(\theta_{d}\right)$ function. Corresponding values of beam divergence $\sigma_{\theta}^{\text {meas }}=5.9 \pm 0.6 \mathrm{mrad}$ and $\sigma_{\theta}^{\text {retr }}=5.2 \pm 0.6 \mathrm{mrad}$.

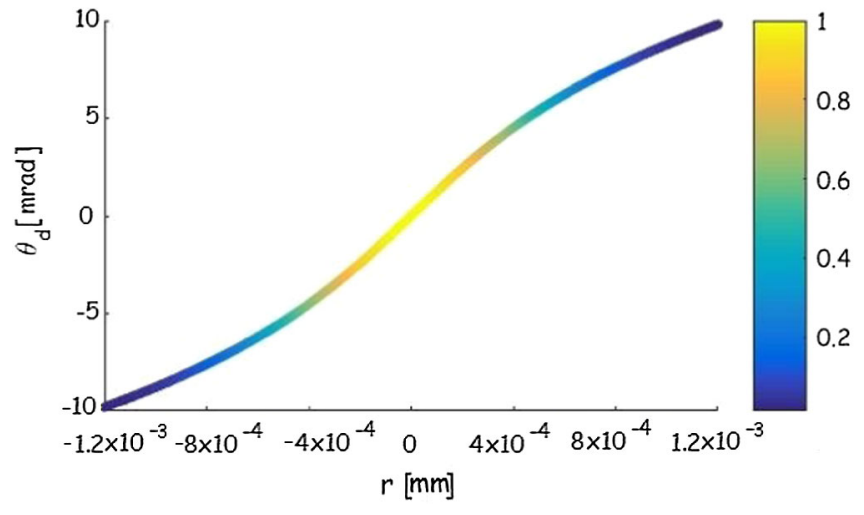

FIG. 7. Reconstructed trace-space density, corresponding to the contour line of the curve $\left\{r, \theta_{d}(r), P(r)\right\}$, where $P(r)$ corresponds to the solid blue line of Fig. (2).

the acceleration length $L_{a c c}$ was almost comparable with the depletion length of the laser $\sim 1.18 \mathrm{~mm}$ and from the angular distribution of the betatron radiation we could expect radial symmetry of the electron beam divergence. Therefore we compared the $\Theta\left(\theta_{d}\right)$ distribution retrieved from the betatron radiation analysis and the electron divergence measured on the scintillator screen of the magnetic spectrometer. The two distributions appear only slightly different. This difference is mostly due to the fact that we are using a simplified model of electron trajectories and correlation function. Nevertheless they present comparable values of rms beam divergence $\sigma_{\theta}$. In fact $\sigma_{\theta}^{\text {meas }}=5.9 \pm 0.6 \mathrm{mrad}$, while $\sigma_{\theta}^{\text {retr }}=5.2 \pm 0.6 \mathrm{mrad}$.

The reconstructed trace-space density is represented in Fig. 7, corresponding to the contour line of the curve $\left\{r, \theta_{d}(r), P(r)\right\}$, where $P(r)$ corresponds the solid blue line of Fig. (2). The measured normalized emittance [40] of the electron beam under consideration comes to be $\epsilon_{r_{\beta} N}=\gamma_{0} \sqrt{\left(\sigma_{\gamma} / \gamma_{0}\right)^{2} \sigma_{r}^{2} \sigma_{\theta}^{2}+\epsilon_{r_{\beta}}^{2}}=(0.6 \pm 0.1) \mathrm{mmmrad}$.

\section{CONCLUSIONS}

It is worth noting that estimating the emittance as $\gamma_{0} \sigma_{r} \sigma_{\theta}^{\text {meas }}$ yields $\epsilon_{r_{\beta} N}^{\text {no-corr }}=(1.5 \pm 0.3) \mathrm{mm} \mathrm{mrad}$, which is quite larger than the value obtained from a complete analysis including the correlation term. Therefore, in order to accurately measure the beam emittance inside the plasma bubble, the correlation term is necessary. For any application requiring the capture, via conventional magnets or plasma optics [41], and the utilization of a plasma accelerated electron beam, the knowledge of the electron trace space is fundamental for matching issues. Accurate, high resolution, nonintercepting emittance diagnostic techniques, like the betatron radiation actually can provide, seem to be the most promising for this aim. In the present work a methodology to reconstruct the trace space of plasma accelerated electron beams has been presented. It is shown that the single shot measurement of both the 
betatron radiation spectrum and the electron energy spectrum can provide all the information about the trace space of the electron beam. A direct technique to measure the betatron oscillation amplitude distribution has been described and applied to experimental data, from which the beam transverse profile has been retrieved. From the study of the electron transverse dynamics inside the plasma bubble, the correlation between the betatron amplitude and the divergence, i.e. the angle with respect the acceleration axis, has been found. The distribution of the electron divergences inside the bubble has been retrieved, which is comparable to the one measured outside the plasma when plasma-vacuum interface and space-charge effects are negligible. The knowledge of the trace-space density allows a more accurate measurement of the transverse emittance.

\section{ACKNOWLEDGMENTS}

This work has been partially supported by the EU Commission in the Seventh Framework Program, Grant Agreement No. 312453 EuCard-2, by the Italian Ministry of Research in the framework of FIRB-Fondo per gli Investimenti della Ricerca di Base, Project No. RBFR12NK5K.

[1] A. Rousse, K. Ta Phuoc, R. Shah, A. Pukhov, E. Lefebvre, V. Malka, S. Kiselev, F. Burgy, J. Rousseau, D. Umstadter, and D. Hulin, Production of a keV X-ray Beam from Synchrotron Radiation in Relativistic Laser-Plasma Interaction, Phys. Rev. Lett. 93, 135005 (2004).

[2] S. Wang, C. E. Clayton, B. E. Blue, E. S. Dodd, K. A. Marsh, W. B. Mori, C. Joshi, S. Lee, P. Muggli, T. Katsouleas, F. J. Decker, M. J. Hogan, R. H. Iverson, P. Raimondi, D. Walz, R. Siemann, and R. Assmann, X-ray Emission from Betatron Motion in a Plasma Wiggler, Phys. Rev. Lett. 88, 135004 (2002).

[3] T. Tajima and J. M. Dawson, Laser Electron Accelerator, Phys. Rev. Lett. 43, 267 (1979).

[4] E. Esarey, C. B. Schroeder, and W. P. Leemans, Physics of laser-driven plasma-based electron accelerators, Rev. Mod. Phys. 81, 1229 (2009).

[5] V. Malka, J. Faure, Y. A. Gauduel, E. Lefebvre, A. Rousse, and K. Ta Phuoc, Principles and applications of compact laser-plasma accelerators, Nat. Phys. 4, 447 (2008).

[6] S. Kneip, C. McGuffey, F. Dollar, M. S. Bloom, V. Chvykov, G. Kalintchenko, K. Krushelnick, A. Maksimchuk, S. P. D. Mangles, T. Matsuoka, Z. Najmudin, C. A. J. Palmer, J. Schreiber, W. Schumaker, A. G. R. Thomas, and V. Yanovsky, $\mathrm{X}$-ray phase contrast imaging of biological specimens with femtosecond pulses of betatron radiation from a compact laser-plasma wakefield accelerator, Appl. Phys. Lett. 99, 093701 (2011).

[7] S. Fourmaux, S. Corde, K. Ta Phuoc, P. Lassonde, G. Lebrun, S. Payeur, F. Martin, S. Sebban, V. Malka, A. Rousse, and J.C. Kieffer, Single shot phase contrast imaging using laser-produced betatron x-ray beams, Opt. Lett. 36, 2426 (2011).
[8] S. Kneip et al., Bright spatially coherent synchrotron x rays from a tabletop source, Nat. Phys. 6, 980 (2010).

[9] M. Mo, S. Fourmaux, A. Ali, P. Lassonde, J. Kieffer, and R. Fedosejevs, Characterization of laser wakefield generated betatron $\mathrm{x}$-ray radiation using grazing incidence mirror reflection, Rev. Sci. Instrum. 84, 123106 (2013).

[10] S. Kneip, C. McGuffey, J. L. Martins, M. S. Bloom, V. Chvykov, F. Dollar, R. Fonseca, S. Jolly, G. Kalintchenko, K. Krushelnick, A. Maksimchuk, S. P. D. Mangles, Z. Najmudin, C. A. J. Palmer, K. Ta Phuoc, W. Schumaker, L. O. Silva, J. Vieira, V. Yanovsky, and A. G. R. Thomas, Characterization of transverse beam emittance of electrons from a laser-plasma wakefield accelerator in the bubble regime using betatron x-ray radiation, Phys. Rev. ST Accel. Beams, 15, 021302 (2012).

[11] G. R. Plateau, C. G. R. Geddes, D. B. Thorn, M. Chen, C. Benedetti, E. Esarey, A. J. Gonsalves, N. H. Matlis, K. Nakamura, C. B. Schroeder, S. Shiraishi, T. Sokollik, J. van Tilborg, Cs. Toth, S. Trotsenko, T. S. Kim, M. Battaglia, Th. Stöhlker, and W. P. Leemans, Low-Emittance Electron Bunches from a Laser-Plasma Accelerator Measured using Single-Shot X-Ray Spectroscopy, Phys. Rev. Lett. 109, 064802 (2012).

[12] M. Schnell, A. Sävert, B. Landgraf, M. Reuter, M. Nicolai, O. Jäckel, C. Peth, T. Thiele, O. Jansen, A. Pukhov, O. Willi, M. C. Kaluza, and C. Spielmann, Deducing the Electron-Beam Diameter in a Laser-Plasma Accelerator Using X-Ray Betatron Radiation, Phys. Rev. Lett. 108, 075001 (2012).

[13] A. Köhler, J. P. Couperus, O. Zarinia, A. Jochmann, A. Irman, and U. Schramm, Single-shot betatron source size measurement from a laser-wakefield accelerator, Nucl. Instrum. Methods Phys. Res., Sect. A 829, 265 (2016).

[14] F. Albert, R. Shah, K. Ta Phuoc, R. Fitour, F. Burgy, J. Rousseau, A. Tafzi, D. Douillet, T. Lefrou, and A. Rousse, Betatron oscillations of electrons accelerated in laser wakefields characterized by spectral x-ray analysis, Phys. Rev. E 77, 056402 (2008).

[15] D. B. Thorn, C. G. R. Geddes, N. H. Matlis, G. R. Plateau, E. H. Esarey, M. Battaglia, C. B. Schroeder, S. Shiraishi, Th. Stöhlker, C. Tóth, and W. P. Leemans, Spectroscopy of betatron radiation emitted from laser-produced wakefield accelerated electrons, Rev. Sci. Instrum. 81, 10E325 (2010).

[16] S. Fourmaux, S. Corde, K. Ta Phuoc, P. M. Leguay, S. Payeur, P. Lassonde, S. Gnedyuk, G. Lebrun, C. Fourment, and V. Malka, Demonstration of the synchrotron-type spectrum of laser-produced betatron radiation, New J. Phys. 13, 033017 (2011).

[17] A. Pukhov, S. Gordienko, S. Kiselev, and I. Kostyukov, The bubble regime of laser-plasma acceleration: Monoenergetic electrons and the scalability, Plasma Phys. Controlled Fusion 46, B179 (2004).

[18] W. Lu, M. Tzoufras, C. Joshi, F. S. Tsung, W. B. Mori, J. Vieira, R. A. Fonseca, and L. O. Silva, Generating multi$\mathrm{GeV}$ electron bunches using single stage laser wakefield acceleration in a 3D nonlinear regime, Phys. Rev. ST Accel. Beams 10, 061301 (2007).

[19] M. Ferrario et al., SPARC-LAB present and future, Nucl. Instrum. Methods Phys. Res., Sect. B 183, 309 (2013). 
[20] D. Giulietti, The particle laser-plasma acceleration in Italy, J. Phys. Conf. Ser. 508, 012001 (2014).

[21] A. Curcio and D. Giulietti, Innovative $\mathrm{x}-\gamma$ ray sources based on laser-produced plasmas, Nucl. Instrum. Methods Phys. Res., Sect. B 355, 214 (2015).

[22] V. Shpakov, M. P. Anania, A. Biagioni, E. Chiadroni, A. Cianchi, A. Curcio, S. Dabagov, M. Ferrario, F. Filippi, A. Marocchino, B. Paroli, R. Pompili, A. R. Rossi, and A. Zigler, Betatron radiation based diagnostics for plasma wakefield accelerated electron beams at the SPARC-LAB test facility, Nucl. Instrum. Methods Phys. Res., Sect. A 829, 330 (2016).

[23] S. Corde, K. Ta Phuoc, G. Lambert, R. Fitour, V. Malka, A. Rousse, A. Beck, and E. Lefebvre, Femtosecond x rays from laser-plasma accelerators, Rev. Mod. Phys. 85, 1 (2013).

[24] E. Esarey, B. A. Shadwick, P. Catravas, and W. P. Leemans, Synchrotron radiation from electron beams in plasmafocusing channels, Phys. Rev. E 65, 056505 (2002).

[25] A. Pukhov and J. Meyer-ter-Vehn, Laser wakefield acceleration: The highly nonlinear broken-wave regime, Appl. Phys. B 74, 355 (2002).

[26] A. Curcio, D. Giulietti, G. Dattoli, and M. Ferrario, Resonant interaction between laser and electrons undergoing betatron oscillations in the bubble regime, J. Plasma Phys. 81, 495810513 (2015).

[27] L. E. Collins and P. T. Stroud, Extraction of high current ion beams with low divergence, Nucl. Instrum. Methods 26, 157 (1964).

[28] Y. Yamazaki, T. Kurihara, H. Kobayashi, I. Sato, and A. Asami, High-precision pepper-pot technique for a lowemittance electron beam, Nucl. Instrum. Methods Phys. Res., Sect. A 322, 139 (1992).

[29] S. Fritzler, E. Lefebvre, V. Malka, F. Burgy, A. E. Dangor, K. Krushelnick, S. P. D. Mangles, Z. Najmudin, J.-P. Rousseau, and B. Walton, Emittance Measurements of a Laser-Wakefield-Accelerated Electron Beam, Phys. Rev. Lett. 92, 165006 (2004).

[30] E. Brunetti, R. P. Shanks, G. G. Manahan, M. R. Islam, B. Ersfeld, M. P. Anania, S. Cipiccia, R. C. Issac, G. Raj, G. Vieux, G. H. Welsh, S. M. Wiggins, and D. A. Jaroszynski, Low Emittance, High Brilliance Relativistic Electron Beams from a Laser-Plasma Accelerator, Phys. Rev. Lett. 105, 215007 (2010).
[31] C. M. Sears, A. Buck, K. Schmid, J. Mikhailova, F. Krausz, and L. Veisz, Emittance and divergence of laser wakefield accelerated electrons, Phys. Rev. ST Accel. Beams 13, 092803 (2010).

[32] A. G. R. Thomas, Scalings for radiation from plasma bubbles, Phys. Plasmas 17, 056708 (2010).

[33] John David Jackson, Classical Electrodynamics (Wiley, New York, 1999).

[34] L. A. Gizzi, C. Benedetti, C. A. Cecchetti, G. Di Pirro, A. Gamucci, G. Gatti, and T. Levatoy, Laser-plasma acceleration with FLAME and ILIL ultraintense lasers, Applied plant science 3, 559 (2013).

[35] T. Hosokai, K. Kinoshita, T. Ohkubo, A. Maekawa, M. Uesaka, A. Zhidkov, and S. V. Bulanov, Observation of strong correlation between quasimonoenergetic electron beam generation by laser wakefield and laser guiding inside a preplasma cavity, Phys. Rev. E 73, 036407 (2006).

[36] A. Curcio, M. Anania, F. G. Bisesto, A. Faenov, M. Ferrario, M. Galletti, D. Giulietti, R. Kodama, M. Petrarca, T. Pikuz, and A. Zigler, Characterization of X-ray radiation from solid Sn target irradiated by femtosecond laser pulses in the presence of air plasma spark, Laser Part. Beams 34, 533 (2016).

[37] S. Sinigardi, A. Marocchino, P. Londrillo, and A. Sgattoni, ALaDyn v1.0.0-beta, http://doi.org/10.5281/zenodo.49553 (2016).

[38] C. Benedetti, A. Sgattoni, G. Turchetti, and P. Londrillo, A high-accuracy PIC Code for the Maxwell-Vlasov equations, IEEE Trans. Plasma Sci. 36, 1790 (2008).

[39] P. Londrillo, C. Benedetti, A. Sgattoni, and G. Turchetti, Charge preserving high order PIC schemes, Nucl. Instrum. Methods Phys. Res., Sect. A 620, 28 (2010).

[40] M. Migliorati, A. Bacci, C. Benedetti, E. Chiadroni, M. Ferrario, A. Mostacci, L. Palumbo, A. R. Rossi, L. Serafini, and P. Antici, Intrinsic normalized emittance growth in laser-driven electron accelerators, Phys. Rev. ST Accel. Beams 16, 011302 (2013).

[41] J. van Tilborg, S. Steinke, C. G. R. Geddes, N. H. Matlis, B. H. Shaw, A. J. Gonsalves, J. V. Huijts, K. Nakamura, J. Daniels, C. B. Schroeder, C. Benedetti, E. Esarey, S. S. Bulanov, N. A. Bobrova, P. V. Sasorov, and W. P. Leemans, Active Plasma Lensing for Relativistic Laser-PlasmaAccelerated Electron Beams, Phys. Rev. Lett. 115, 184802 (2015). 